\title{
Interactive Technologies for Children with Special Needs
}

\author{
Meryl Alper \\ Annenberg School for \\ Communication \& Journalism \\ University of Southern California \\ Los Angeles, CA 90089 USA \\ malper@usc.edu
}

\author{
Juan Pablo Hourcade \\ Department of Computer Science \\ University of lowa \\ lowa City, IA 52242 USA \\ juanpablo- \\ hourcade@uiowa.edu
}

\author{
Shuli Gilutz \\ Sammy Ofer School of \\ Communication \\ Interdisciplinary Center (IDC) \\ Herzliya, 46150 Israel \\ shulig@idc.ac.il
}

\begin{abstract}
There is a growing trend in both the academic and private sectors of designing innovative interactive technologies for children. These technologies could be a unique platform for addressing the freedoms and rights of children with special needs. They afford new kinds of support for children with special needs' full participation, both as children and later on as adults, in the public sphere. This workshop highlighted three underexplored themes in designing interactive technologies for children with disabilities, including considerations for participatory design, interactive technologies for children with hearing impairments, and the possibly transformative potential of tangible computing. Some of the future research opportunities and challenges in the areas of deep engagement, interdisciplinarity, individuality, and practicality are discussed.
\end{abstract}

\section{Categories and Subject Descriptors}

H.5.2 [Information Interfaces and Presentation]: User Interfaces; K.4.2: [Computers and Society]: Social Issues Assistive technologies for persons with disabilities

\section{General Terms}

Human Factors

\section{Keywords}

Children, disabilities, hearing impairment, participatory design, special needs, tangible computing, children's HCI.

\section{INTRODUCTION AND BACKGROUND}

Individual freedoms at the core of modern democratic systems have brought unprecedented quality of life to their citizens. In 1941, at a time when these ideas were being directly challenged, United States President Franklin D. Roosevelt spoke of four universal freedoms: freedom of speech and expression, freedom of worship, freedom from want, and freedom from fear. For people with disabilities and special needs, attaining these freedoms - in particular the first and third - can be particularly challenging. This is especially true as we enter an age where our

Permission to make digital or hard copies of all or part of this work for personal or classroom use is granted without fee provided that copies are not made or distributed for profit or commercial advantage and that copies bear this notice and the full citation on the first page. To copy otherwise, or republish, to post on servers or to redistribute to lists, requires prior specific permission and/or a fee.

IDC'12, June 12-15, 2012, Bremen, Germany.

Copyright 2012 ACM ability to interact with computers plays a significant role in how successfully we can express our opinions, stay informed, participate in civic life, and fulfill many of our daily pursuits, from leisure activities to using public transportation.

Growing up with a special need or disability only makes these challenges steeper, though not insurmountable. All children need to grow up experiencing the world, interacting with peers, family and adult caregivers, and developing the skills that can lead to independent life in adulthood. This may be more of a challenge to children with special needs, as the environment around them is not always best designed for their learning needs. Interactive technologies can play a positive role in helping children manage these challenges, from communicating with others, to learning in school, to experiencing and enjoying the world.

An increasing number of academic researchers have been answering the call to design these technologies, and to investigate how mainstream technologies could better suit children with disabilities. This call comes with additional challenges. For example, there are diverse stakeholders that need to be involved in the design and adoption of these technologies, from the children themselves, to their families, and various adult caregivers. Likewise, researchers and practitioners with a variety of expertise need to work together to develop practical solutions with a chance of succeeding in real-world environments. Finally, technology designers need to consider the individual realities of children and how these may change and evolve, from their own needs and abilities, to their media and technology ecology, to the people (including other children) with whom they will be learning, playing, and communicating.

In the following sections we describe the specific contributions that were presented at the Interactive Technologies for Children with Special Needs workshop held at the Interaction Design and Children 2012 conference. We follow this by a discussion of these contributions and additional challenges based on key principles.

\section{POSITION PAPER CONTRIBUTIONS}

In this section, we summarize the workshop submission papers. We organized these contributions into three themes. The majority of contributions centered around the first theme of participatory design, though each paper identified a different range of potential "participants" involved in the participatory research process. The second theme, interactive technologies for children with hearing impairments, includes contributions offering best practices for designing assistive technologies for this population within both mainstream and special education classrooms. The third theme, tangible computing, includes contributions discussing how different aspects of tangible toy systems can support independent and collaborative play. 


\subsection{Participatory Design}

\subsubsection{Key Challenges and Opportunities}

Frauenberger, Good \& Alcorn propose key challenges, opportunities, and potential future research agendas in designing interactive technologies for children across various disabilities. They ground their discussion in their experiences developing ECHOES, a technology-enhanced shared learning environment for both children with autism spectrum conditions and their typically developing peers. The researchers identify various meso- and macro-level challenges and opportunities for implementing participatory design approaches specific to children with disabilities as a population, yet generalizable across children's different abilities, needs, and conditions. These mesolevel issues include developing relationships among stakeholders; facilitating communication that may require communicative aids; negotiating structured and unstructured creative prompts for children with particular disabilities; mindful interpretation and translation of the input generated by children with disabilities; and low levels of acceptance of "messy" qualitative research in the mainstream scientific community. In addition, the researchers identified two macro-level issues concerning the implications of participatory design with children with disabilities. These include the relationship between digital inclusion and social inclusion, and tensions between those who accept or reject person- and childfirst language, or those who use such terminology interchangeably.

\subsubsection{Sociological Approaches to Inclusive Play and Participatory Design Processes}

Holt conducts interdisciplinary research on inclusive play between disabled and non-disabled children. The paper reflects on the moral assumptions and potential prejudices underlying philosophies of inclusive design and education. It describes the early stages of the action-research project, Together Through Play. This UK-based initiative by engineers and sociologists will explore disabled and non-disabled children's aspirations for collaborative play and how the design of toys, games, and play environments can support or hinder meaningful play. The project will attempt to untangle the notion of "inclusion" in a more holistic manner, acknowledging and problematizing the social, political and cultural contexts, which influence technological design practices. The author's theoretical approach isolates access factors, including contact or proximity within classroom environments, from those of social and emotional factors of inclusion. Holt hypothesizes that these interpersonal factors, if understood through participatory design processes, have the potential to maximize the accessibility of physical and virtual environments, and foster positive interactions and relationships between disabled and non-disabled children.

\subsubsection{Interactive Model for Collaborative Design of an Intelligent Playground}

Helms is working on I-Space, an intelligent physical playground with embedded computer games. The playground combines IT systems, sensor technologies, and training equipment. I-Space registers, collects, and compares data on patients, enabling physical therapists and social workers to monitors their clients' progress. The playground is designed for both children and adults, recognizing all users as citizens with equal rights. The goal of the project is to enhance learning and motivation for users through participating in the design process and using the final product. The author proposes a "quadrant model" of participatory design and multi-disciplinary collaboration. This four-stage process for scaffolding participatory design, combines different theoretical traditions of user-driven innovation and design into a generalizable model. In order, the stages are: 1) observing; 2) constructing; 3) co-constructing; and 4) re-constructing. Issues for discussion include supporting ongoing reflection on open and closed design processes; the temporary or permanent nature of research environments; the importance of flexible prototypes; and the role of student researchers.

\subsubsection{Longitudinal Research in Classrooms with Special Educational Needs}

Williams, Meckin, Bryan-Kinns \& Stockman are conducting longitudinal participatory design research at a special needs school in the UK. The authors suggest two main considerations for researchers to take into account when conducting collaborative research embedded in special education classrooms. Their first suggestion is that researchers consider the contextual factors of working in diverse and busy school environments, including gaining initial and sustained access to participants, syncing school and university academic calendars, and the diverse behavioral traits exhibited by the participants that may vary when they are with teachers or researchers on any given day. The authors' second suggestion for researchers is to maintain and repair relationships with stakeholders. This 'repair work' may include clearly communicating when researchers will and will not be at the school, and researchers adding value to and building capacity within classrooms. The authors conclude by emphasizing that while embedded longitudinal participatory design research in special needs classrooms may be demanding at times, the pursuit is richly rewarding, exciting, and enjoyable.

\subsubsection{Parents as Participants}

Pihlainen-Bednarik discusses ongoing longitudinal research into the under-researched area of including parents in the participatory design, use, and evaluation of interactive technologies. This paper is based on the researcher's experience in Finland from 20092012 in technology clubs for children with special needs and their parents. The children in these clubs are ages $4-13$, include both boys and girls, and are diagnosed as having physical (cerebral palsy) or learning difficulties (autism spectrum disorders, developmental delays, and/or difficulties in academic and life skills, such as concentration and communication.) The author describes three primary challenges in including parents in the participatory design, use, and evaluation of interactive technologies. These three areas are motivation, organization, and interaction. In terms of motivation, challenges include convincing parents of the benefits of participation in the project, as well as helping parents gain familiarity with potentially new technologies and methodologies. To meet this challenge of motivation, the author suggests that future researchers dedicate enough time for briefing and discussions with parents before starting a participatory design project. In terms of organization, challenges include syncing the schedules of researchers, parents, and children. To meet this challenge, the author suggests that researchers be mindful of family free time and parent work obligations. In terms of interaction, challenges include the potential for unequal distribution of power and decision-making. To meet this challenge, the author suggests that researchers be reflective of privileging their kind of expert knowledge over parents' expertise. The author also suggests open, transparent, and visible documentation of research processes for parents. 


\subsubsection{Cultivating Empathy Among Research Partners with Different Abilities}

Duysburgh, Slegers \& Jacobs describe their process in designing interactive applications for children with hearing impairments in Belgium. The authors describe the three phases of their research: inspiration, ideation, and conceptualization. In the inspiration phase, researchers conducted a qualitative ethnographic mapping of experiences of children with hearing impairment. For the ideation phase, the researchers developed two workshops with the design team: an empathizing workshop and a brainstorm workshop. In the empathizing workshop, the researchers raised concerns about the authenticity and the reinterpretation of data from children with hearing impairments by sign language interpreters, field researchers, and the rest of the design team. The brainstorm workshops resulted in one concept to be developed further, a device that improves and facilitates reading comprehension of hearing-impaired children by making use of rich annotated texts. The reading comprehension device developed through the inspiration, ideation, and conceptualization phases is currently under development in a follow-up research project.

\subsection{Interactive Technologies for Children with Hearing Impairments}

\subsubsection{Recommendations for Participatory Design with Children with Hearing Impairments}

Duysburgh, Slegers \& Jacobs concluded their paper with a series of recommendations for research teams planning to involve children with hearing impairments in a participatory design process focusing on interactive technologies. The researchers made five recommendations. The first recommendation is that researchers be aware of the diversity of opinions about hearing impairment by stakeholders and organizations. The second recommendation is that researchers be aware of some groups' hesitance to become involved with the project out of a valid fear of exploitation of children with hearing impairments. The third recommendation is that the inspiration, ideation, and conceptualization phases incorporate dynamic feedback between the design team, stakeholders, and the target group. The fourth recommendation is to be cognizant of the potential reinterpretation and distortion of input directly from children with hearing impairment. Lastly, the researchers recommended that researchers developing technologies for children with hearing impairments actively look for novel ways to engage the target group, no matter how challenging.

\subsubsection{Virtual Reality to Support Communicative and Cognitive Development}

Eden discusses the results of several studies exploring the benefit of immersive $3 \mathrm{D}$ virtual reality technology in the teaching and treatment process of children with hearing impairment. These studies focused on enhancing children's cognitive and language abilities. The research focused on improving two skills: time sequence perception and induction. In terms of time sequence perception, findings suggest that virtual reality representation improves time sequence perception for children ages 4-10, including children with and without hearing impairment, more so than pictorial, textual, aural, and signed representation. The authors suggests with caution that young deaf and hard-of-hearing children's accomplishments in sequencing tasks are dependent on the mode of representation. In terms of inductive processes, findings suggest that practicing with 3D spatial rotations in a VR Tetris game significantly improved inductive thinking for an experimental group of children with hearing impairment, as compared with the control group of children with hearing impairment who played a similar 2D Tetris game. Moreover, the children with hearing impairment who played a 3D VR Tetris game had improved inductive abilities to the extent that there was no noticeable difference between them and another control group of hearing children who received no intervention.

\subsection{Tangible Computing}

\subsubsection{Supporting Independent Exploration for Students with Intellectual Disabilities}

Pontual and Price investigate how different aspects of tangible computing, across a range of different tangible systems, can better support more independent exploration and experimentation for heterogeneous school groups of students with intellectual disabilities. The researchers explored the following tangible systems: D-touch drum machine, Sifteo cubes, a tangible interactive tabletop, an object augmented with LEDs, and an accelerometer. Three relevant themes have emerged from ongoing research. One theme is the importance of both space and time dimensions of embodiment. Another theme is the challenges of conveying concepts through audio representations. Lastly, the authors describe the emergent theme of the role of free and constrained actions as cognitive resources for thinking and expression.

\subsubsection{Encouraging Collaborative Play Among Children with Autism in India}

Dsouza, Barretto \& Raman are exploring the design and impact of a set of interactive multi-sensory tangible toys on social and collaborative play among 14 children age 5-11 at a school for children with autism spectrum disorders in India. The tangibles, a set of toys named Uncommon Sense, are shared objects that children must use collaboratively in order to trigger multi-sensory feedback from the system. A child's actions, voice, and movements can trigger visual, aural, and tactile feedback from the toys. Each toy has cause-effect behavior that can be triggered by either individual or collaborative play. The toys have no recognizable shape as to avoid any prior meanings potentially associated with them by autistic and non-autistic users. The research is being conducted with three types of Uncommon Sense toys, each focusing on a play experience: orientation, touch, and expression. Analysis of data thus far indicates that the sensory feedback of the tangibles facilitates the development of various play skills such as joint attention, imitative social interaction, and turn taking.

\section{DISCUSSION}

As the Interaction Design and Children community increases the amount of research on designing technologies for children with special needs, it is important to think about the factors that can make these pursuits successful. Based on a reflection on his previous research, one of the co-organizers of the workshop (Hourcade) has proposed four principles to consider when designing technologies for populations with special needs: deep engagement, interdisciplinarity, individuality, and practicality. Below, we discuss each principle and how it is reflected in the workshop. 


\subsection{Deep Engagement}

Participatory design, and partnerships with children and other stakeholders have long had a strong presence in the interaction design and children community [1][2]. The conference regularly features novel techniques for involving children in the design process. With children with special needs though, the need for deep engagement with all stakeholders, and with the children's needs becomes even more important.

One reason is that children with special needs will be different from most designers and developers' own childhood experiences, or children they know. It is also the case that there tend to be many more stakeholders who should have a voice in the design process. This includes parents, siblings, teachers, special education staff, and therapists. Technologies developed for children with special needs need to work in the context of their daily interactions, with all these important people in their lives. Additionally, success or failure will also depend on how well these technologies match children's social, physical and environmental requirements.

Children with special needs are also difficult to access, simply because they are few in number. This also requires a deep engagement with the population, including parent support groups and other community organizations. These ties can facilitate access to the children and at the same time make it more likely that the technologies developed will have a positive impact.

Finally, deep engagement is also necessary in getting to know what makes the target population different from typically developing children, with extra efforts in reviewing literature and collaborating with experts necessary. Not surprisingly, most of the position statements submitted to this workshop discuss efforts and benefits of involving children with special needs and stakeholders in the design process.

\subsection{Interdisciplinarity}

Typical academic projects on designing technologies for children usually do not include experts on human development, although researchers often have at least some background in cognitive development. When it comes to children with special needs though, there is a strong need for wider sets of expertise. This is true both in terms of better understanding the needs and abilities of children, but sometimes also in terms of building technologies with innovative forms of interaction. Scholars from areas such as communication, occupational therapy, developmental psychology, special education, and learning sciences have unique contributions to make at various stages. This expertise is key in building interactive technologies that are tied to different content areas (e.g., science and math, music composition.)

\subsection{Individuality}

A significant difficulty in designing for children with special needs is the great variability within these populations. Some children with disabilities will display significantly different behaviors based on the context they are in and the medications they are taking. Multiple disabilities can add even more complexity. Gifted students with learning differences also need to be taken into account. Across cultures and countries, norms around parent involvement may differ. For all these reasons, interactive technologies for children with special needs need to address individual needs and avoid one-size-fits-all approaches. In the future, Universal Design for Learning principles [3] could also be incorporated into the research and design process.

\subsection{Practicality}

A rewarding aspect of designing interactive technologies for children with special needs is the potential of having a direct, positive impact on the children. At the same time, because these children have greater needs than typically developing children, we have the responsibility of designing technologies that can reasonably work in their real-world environments, and that one day could be adequately priced so that at least a majority of families can afford them. As researchers, we need to go beyond developing novel systems to be tested in a lab so we can get publications and grants. For this population in particular, we need to make an effort to develop technologies that can quickly be used to help children outside of our research programs. A practical system ready for the real world is going to have a greater impact than a state-of-the-art setup that will never leave the lab.

Children use technology not only at school, but also at home and other out-of-school contexts. Future research should take into account leisure activities such as video gaming, which may support motivation and enjoyment in children with special needs. Young people with disabilities use a range of assistive technologies in their daily lives (e.g., wheelchairs, robotic aids for daily living.) Children with disabilities might also use their favorite non-digital props, toys, and stuffed animals alongside interactive technologies. Researchers and designers should take into account the full media ecosystem of children with disabilities.

\section{CONCLUSION}

Academic researchers do not lay sole claim to designing technologies for children with disabilities. The growth of private sector ventures, particularly iPad apps for children with disabilities, an increasingly complex media ecology for children with special needs, and potential digital divides across class, ethnicity, and geography necessitate a more public dialogue about the themes, challenges, and opportunities this workshop raises. The three position paper themes highlighted include considerations for participatory design, interactive technologies for children with hearing impairments, and innovations in tangible computing. When designing technologies for and with children with special needs, the principles of deep engagement, interdisciplinarity, individuality, and practicality, should be reflected upon at the projects' inception and throughout its development cycle.

\section{ACKNOWLEDGMENTS}

Thank you to the researchers who participated in this workshop.

\section{REFERENCES}

[1] Druin, A. 2002. The role of children in the design of new technology. Behaviour and Information Technology, 21(1):1-25.

[2] Guha, M. L., Druin, A., and Fails, J.A. 2008. Designing with and for children with special needs: an inclusionary model. In IDC '08 Proceedings of the 7th International Conference on Interaction Design and Children, 61-64, New York, NY, USA: ACM

[3] Rose, D. H., and Meyer, A. (Eds.). 2006. A Practical Reader in Universal Design for Learning. Cambridge, MA: Harvard Education Press. 
